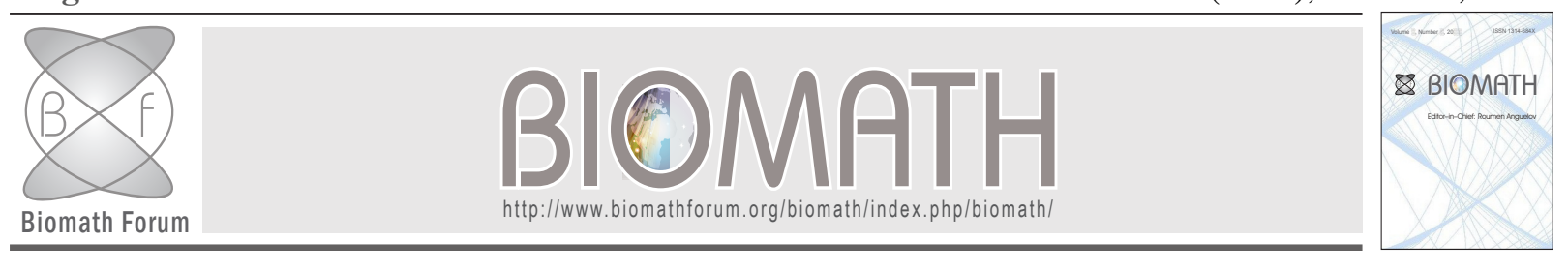

\title{
On the Mathematical Modelling of EPS Production by a Thermophilic Bacterium
}

\author{
Nadja Radchenkova ${ }^{1}$, Margarita Kambourova ${ }^{1}$, Spasen Vassilev ${ }^{1}$, Rene $\mathrm{Alt}^{2}$, Svetoslav Markov ${ }^{3}$ \\ ${ }^{1}$ Institute of Microbiology, Bulgarian Academy of Sciences \\ 2 Sorbonne Universities, LIP6, UPMC, CNRS UMR7606 \\ ${ }^{3}$ Institute for Mathematics and Informatics, Bulgarian Academy of Sciences
}

Received: 24 January 2014, accepted: 12 July 2014, published: 23 July 2014

\begin{abstract}
This paper presents experimental data coming from a batch fermentation process and theoretical models aiming to explain various aspects of these data. The studied process is the production of exopolysaccharides (EPS) by a thermophilic bacterium, Aeribacillus pallidus 418, isolated from the Rupi basin in South-West Bulgaria. The modelling approach chosen here is: first, biochemical reaction schemes are formulated, comprising several reaction steps; then the reaction schemes are translated into systems of ordinary differential equations (ODE) using the mass action law; then the ODE systems are studied by means of numerical simulations. The latter means that the ODE systems are parametrically identified in order to possibly fit the experimental data. A main peculiarity of the proposed reaction schemes, resp. models, is the assumption that the cell biomass consist of two dynamically interacting cell fractions (dividing and non-dividing cells). This assumption allows us to implement certain modelling ideas borrowed from enzyme kinetics. The proposed models are compared to a classical model used as reference. It is demonstrated that the introduction of the two cell fractions allows a much better fit of the experimental data. Moreover, our modelling approach allows to draw conclusions about the underlying biological mechanisms, formulating the
\end{abstract}

latter in the form of simple biochemical reaction steps.

Keywords-batch fermentation processes, thermophilic bacterium, reaction schemes, dynamic models, numerical simulations

\section{INTRODUCTION}

An increasing interest towards microbial exopolysaccharides (EPS) is determined by the wide variety of their properties as a result of diversity in their composition. The biodegradability of EPS has an impact on environmentally friendly processes. Thermophilic microorganisms offer short fermentation processes, better mass transfer, decreased viscosity of synthesized polymer and of the corresponding culture liquid.

EPS from thermophilic microorganisms are of special interest due to the advantages of the thermophilic processes and the non-toxic nature of the polymer allowing applications in food and pharmaceutical industries [4]. Low EPS production in thermophilic processes determines the importance of mass transfer optimization for further development of thermophilic processes in an industrial 
scale.

The aim of the current work is to describe and study the dynamics of microbial growth and product synthesis by means of suitable mathematical models, which may give more understanding for the underlying biochemical mechanisms. Our main assumption from a biological perspective is the existence of two cell fractions: dividing and nondividing cells, as proposed in [10]. Based on comparisons with a classical model it is demonstrated that the introduction of the two cell fractions allows a much better fit of the experimental data. In addition, our modelling approach allows to formulate the underlying biological mechanisms in the form of simple biochemical reaction steps.

To achieve our aim we propose several biochemical reaction schemes that conform with the basic assumption for the existence of two cell fractions. Applying the mass action law the reaction schemes lead to dynamical models that are parametrically identified in order to fit the observed data. We demonstrate that the proposed models are compatible with classical Monod fermentation models which are based on different principles. In order to visualize the numerically computed theoretical results, the latter are graphically compared to experimental data. The graphics show that the proposed models reflect specific features of the mechanism of the fermentation process, which may suggest further experimental and theoretical work. We believe that using the proposed approach one can study the basic mechanisms underlying the dynamics of cell growth, substrate uptake and product synthesis. Finally, we hope that the present study will contribute to the optimization of mass transfer and an enhancement of EPS yield by Aeribacillus pallidus 418.

The paper is structured as follows. The methods for the experiments are described in Section II. Section III presents the experimental results and Section IV outlines the modelling approach. Section $\mathrm{V}$ is devoted to several theoretical models whose solutions fit the experimental measurements. The underlying idea is that such a theo- retical approach can help biologists in choosing or rejecting a possible mechanism for the dynamics of microbial growth and EPS synthesis. The numerical solutions obtained with each proposed model are compared to the experimental data and commented. The conclusion explains the merits of the proposed approach and comments on the suggested biological assumptions. It is demonstrated that the proposed models based on the assumption of existence of two cell fractions (dividing and non-dividing cells) are compatible with classical Monod fermentation models.

\section{MATERiAls And Methods}

\section{A. Strain, medium and cultivation}

Aeribacillus pallidus 418 was isolated from a hot spring at Rupi basin, South-West Bulgaria, and selected as an EPS producer among 38 thermophilic bacterial polymer producers [12].

\section{B. Experimental set-up and operation mode}

A 1.5 L jacketed glass reactor (AK-02, Russia) equipped with a hydrofoil impeller Narcissus (0.05 $\mathrm{m}$ diameter) was filled with $1 \mathrm{~L}$ of MSM and $35 \mathrm{~mL}$ of $18 \mathrm{~h}$ strain culture was added as an inoculum. Fermentation parameters such as temperature, $\mathrm{pH}$, foaming and aeration were kept constant during the whole processes performed. Temperature was $55^{0} \mathrm{C}$; $\mathrm{pH}$ value was maintained at 7.0 due to buffer properties of the medium; air flow $0.8 \mathrm{vvm}$ (volume per volume medium) was continuously supplied.

\section{Determination of growth and EPS production}

The influence of agitation on the growth and EPS production was followed at 100, 400, 500, 600 and $800 \mathrm{rpm}$. Growth was determined by measuring of turbidity at $660 \mathrm{~nm}$. A correlation curve reflecting the proportionality between turbidity and dry weight was obtained for the chosen strain. One unit OD corresponds to $1.05 \mathrm{mg} \mathrm{ml}^{-1}$ dry cells of Aeribacillus pallidus 418. EPS was recovered from the culture supernatant samples as previously described [4]. 


\section{EXPERIMENTAL RESULTS FOR BATCH CULTIVATION}

\section{A. Influence of agitation speed}

Comparison of growth curves at different agitation conditions show that a longest exponential phase $(8 \mathrm{~h})$ and lowest biomass yield reached at stationary phase is observed at a lowest speed of stirring (100 rpm) (Figure 1). Exponential phase duration of $4-5 \mathrm{~h}$ is observed for all other agitation speeds. An increase in biomass accumulation and polymer production is observed with increase of stirring speed being highest at $500 \mathrm{rpm}$ for growth and $600 \mathrm{rpm}$ for EPS, after that it decreased. The lowest speed of stirring (100rpm) unfavorable for growth and EPS production and the speed that provide best growth $(500 \mathrm{rpm})$ and best product synthesis $(600 \mathrm{rpm})$ have been chosen for further modelling.

Investigations on the influence of agitation speed on EPS production (Figure 2) reveal that the observed polymer production is connected with the biomass accumulation. First quantities are registered in the early exponential phase and highest levels in the stationary phase. The concentration of the measured polymer is highest at $600 \mathrm{rpm}$ (124 $\left.\mu \mathrm{g} \mathrm{mL}^{-1}\right)$, it is five fold higher than that at 100 rpm $\left(24 \mu \mathrm{g} \mathrm{mL}^{-1}\right)$.

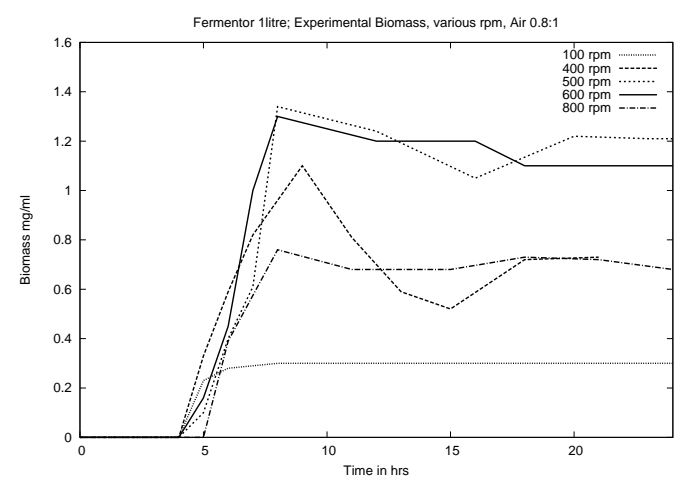

Fig. 1. Experimental biomass for different rpm

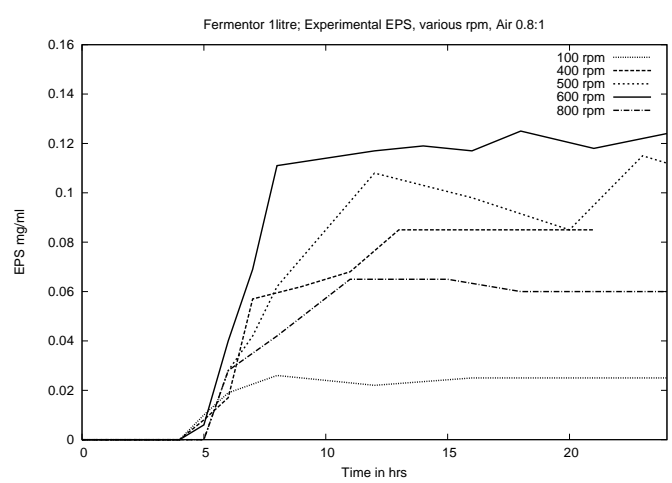

Fig. 2. Experimental EPS for different rpm

The experimentally obtained average values are presented in Figure 1 for the biomass and in Figure 2 for EPS. The figures show clearly that the best production of EPS appears for a speed of 600 revolutions per minute. The experimental results corresponding to maximum EPS yield (at $600 \mathrm{rpm}$ ) are presented in Table II. The measurement error is estimated to be $\leq 15 \%$ for the biomass data ( $\leq 18 \%$ for the last three data) and $\leq 5 \%$ for the EPS data. Taking into account the measurement error, the experimental data of Table 1 are used in the modelling process as interval data [9]. For example, biomass data for hour seven is 1.0 , hence the resp. interval value is $1.0 \pm 15 \%=[0.85,1.15]$. The interval data are visualized as vertical segments in the presented figures.

\begin{tabular}{|l|ccccc|}
\hline Time (hours) & 0 & 1 & 2 & 3 & 4 \\
\hline Biomass $(\mathrm{mg} / \mathrm{ml})$ & 0 & 0 & 0 & 0 & 0 \\
\hline \hline Time (hours) & 5 & 6 & 7 & 8 & 12 \\
\hline Biomass $(\mathrm{mg} / \mathrm{ml})$ & 0.16 & 0.45 & 1.0 & 1.3 & 1.2 \\
EPS $(\mathrm{mg} / \mathrm{ml})$ & 0.006 & 0.037 & 0.07 & 0.112 & 0.117 \\
\hline \hline Time (hours) & 14 & 16 & 18 & 21 & 24 \\
\hline Biomass $(\mathrm{mg} / \mathrm{ml})$ & 1.2 & 1.2 & 1.1 & 1.1 & 1.1 \\
EPS (mg/ml) & 0.119 & 0.120 & 0.124 & 0.124 & 0.124 \\
\hline
\end{tabular}

TABLE I

EXPERIMENTAL VALUES OF THE BIOMASS AND EPS 


\section{MODELLING APPROACH}

The experimental measurements for the cell growth and EPS production together with the estimated measurement errors have been used for testing and studying several dynamical models aiming to explain the nature of the involved metabolic reactions. To this end an original modelling approach similar to the one used in (bio)chemistry has been used. Instead of starting the construction of the model with formulating systems of ordinary differential equations (ODE) and specifying reaction terms in the right-hand side of the system's equations as usually done in classical cell growth models, we start with proposing suitable reaction schemes. The origin of such an approach is seen in the works [2], [6], where reaction steps involving variable reaction rate coefficients are admitted. Our reaction schemes differ from the ones used in these works in that all reaction rate coefficients are numerical constants-as (commonly) in biochemistry and more specifically in Henri-Michaelis-Menten enzyme kinetics [10], [14], [16]. The proposed reaction schemes are then translated into systems of ODE's using the mass action law, and the ODE systems are studied by means of numerical simulations.

Another peculiarity of our modelling approach is the partitioning of the cell biomass into two dynamically interacting fractions of dividing and non-dividing cells. This allows to implement ideas borrowed from enzyme kinetics-there enzymes are subdivided into two fractions: free and bound. Bio-reactor models using fractions (compartments) for the biomass are known in the literature [6], [7], [17]. The proponents of the modelling approach using fractions of the cell biomass note that such structured models allow a better fit of the cell growth dynamics in the lag phase in comparison to the one obtained with classical Monod type models [17]. In our proposed models the cell population is conditionally partitioned into two fractions: dividing and non-dividing cells. This allows to relax the rather restrictive assumption for synchronized states of individual cells that follows from (or is incorporated in) classical models using only one variable for the cell biomass. Thus structured models allow us to assume that cells are not all simultaneously in a specific state-something that is observed in reality.

The terms dividing and non-dividing states refer here to individual cells as opposite to the terms lag, log and stationary phases that refer to the cell community. Classical Monod type models consider a population of a microbial species as being in one of the mentioned phases at any given moment. We consider the dividing cell property as a property of the individual cell which may be inherited from the mother cell [5], [13]. This property may change in time from dividing to non-dividing or vice versa depending on the environmental conditions but also on individual cell properties. Individual cell states are close to the biomass phases but do not coincide. If a cell population has been in a lag or stationary phase for a long time, then most (or all) of the individual cells will be in non-dividing state, conversely, if the cell community is in an established log phase, then most (or all) of the individual cells are in a dividing state. However, at the time when the environmental conditions change (from favorable to unfavorable or vice versa), then individual cells do not change their state simultaneously and there is a time interval when cells of both states coexist. Thus whenever the cell community passes from a lag or stationary phase to a log phase (or conversely) there are cells in dividing state, and others in non-dividing state. As will be demonstrated in this work structured models based on the dividing property effectively contribute to overcoming the deficiency of classical methods to adequately describe the bioprocesses during the intermediate time intervals when biomass phases change; we further refer to this problem as "intermediate-phase-deficiency" problem.

One more peculiarity of our modelling approach is that we fit the theoretical solutions of the proposed models into the interval experimental data (data \pm upper bounds for the estimated measurement errors), which contributes to the model verification process [9]. In other words, our aim 
in the parameter identification process is to make the theoretical solutions pass through the interval experimental data. Appropriate parameters for the various models have been obtained using an optimization method for minimizing the sum of the squared differences between the observed experimental data and the computed theoretical solution. But one cannot assert that these parameters are optimal as there are several local minima and different sets of parameters may lead to almost identical solutions. That is why we chose"manually" appropriate sets of parameters with the additional requirement to make the theoretical solutions pass within as many as possible interval experimental data (measurements plus/minus estimated upper bound for the measurement errors).

\section{MAThematical MODELling}

Several mathematical models are proposed in the sequel and their parameters are numerically identified in order to fit with the observed experimental results as given in Table I] The first model is a classical Monod type model, whereas the other models conform with the idea of a structured bacterial biomass and the reaction-scheme approach as proposed in [10]. Classical Model 1 is used as a reference in order to be compared to the remaining structured models following the reaction-scheme approach.

\section{A. Model 1}

Model 1 is a classical Monod type cell growth model [11]. The model is a modification of a model for CGTase production by bacteria of the species B. circulans ATCC21783 [3]. The model is mathematically represented by the following differential system:

$$
\begin{aligned}
& d x / d t=(\mu-\gamma) x \\
& d s / d t=-(\mu / \delta) x \\
& d p / d t=(\alpha \mu+\beta) x
\end{aligned}
$$

wherein:

$$
\mu(s)=\left(\mu_{\max } s /\left(K_{s}+s+K_{i} s^{2}\right),\right.
$$

with initial conditions $s(0)=s_{0}, \quad x(0)=$ $x_{0}, p(0)=p_{0}$.
Typically model (1) makes use of a growth rate parameter $\mu$ which is not a constant but a function; in this case $\mu$ is a function on the substrate concentration $s$, namely the familiar Andrews/Haldane specific growth rate function $\mu(s)$. The meaning of the numeric parameters in model (1) is given in [3]. Computer simulations provide the following set of parameters: $\mu_{\max }=5, \alpha=93, \beta=$ $0, \gamma=0.005, K_{s}=0.25, K_{i}=30, \delta=13$ with initialization: $x_{0}=0.05, s_{0}=0.1, p_{0}=0$.

The experimental data and the computed theoretical solutions of model (1) are visualized in Figure 3. The vertical intervals correspond to the experimental values as given in Table 1 together with the measurement errors in the data (the same pattern is followed in the remaining figures). The aim has been to identify the parameters of system (1) in such a way that the computed solutions fit the experimental data in the sense that they possibly pass through the experimental interval data.

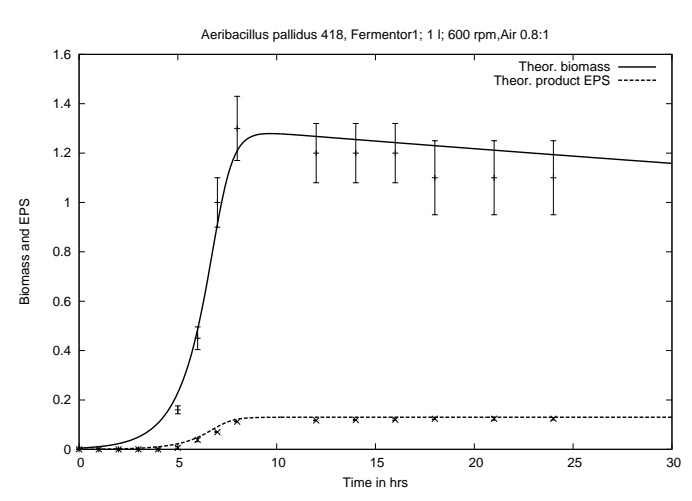

Fig. 3. Solution of model [1], $600 \mathrm{rpm}$

Monod type models describe quite well bioprocesses under favorable conditions and hence allow a good fit of the log (exponential) phase. However, such models do not generally allow good fit for the intermediate phases, e. g. between the 
lag and log phases, as is observed on Fig 3. Note that all experimental values for the biomass in the first four hours are zero whereas the computed theoretical solution for the biomass increases quite rapidly and cannot be fitted well for any values of the model parameters to the experimental interval data for $t \leq 5$. Thus model (1) demonstrates the inherent "intermediate-phase-deficiency" of classical Monod type models.

A characteristic feature of Monod models is the use of the specific growth rate function $\mu=\mu(s)$. Some authors formulate reaction schemes that make use of such a reaction rate function (instead of coefficient!) [2], [6]. It has been demonstrated that Monod type models cannot be induced from reaction schemes with constant reaction rate coefficients by means of the mass action law [10]. It has been also discussed in [10] that Monod type models are special cases of appropriate structured models under specific restrictions (providing exponential growth).

In order to overcome the "intermediate-phasedeficiency" problem and to obtain a better fit for the experimental interval data two original models are proposed and numerically tested using the same experimental data. These models follow the modelling approach of building biochemical reaction schemes with constant reaction rate coefficients [10]. The proposed models are expected to throw some light on the related cell growth mechanisms; both proposed models are based on the idea of structured biomass and the use of an enzyme-kinetics-like reaction scheme as a starting point.

\section{B. Model 2}

Here the total biomass has been split into two fractions of non-dividing $X$-cells and dividing $Y$ cells. According to the reaction-scheme modelling methodology we first formulate suitable reaction steps underlying the biological (biochemical) mechanism behind the cell growth and cell production processes. The corresponding reaction scheme involves six reaction steps: a double growth step (RSG), two reproduction steps (RSR), a producing step (RSP) and a decay step (RSD). As shown in [10] the growth step (RSG) reflects the transition of $X$-cells into $Y$-cells and substitutes the use of the "specific growth rate function" $\mu(s)$ proposed by Monod [11]. This step also reflects the conversion of nutrient substrate $S$ into metabolic products $P$ used for the growth of the cells and for their preparation to pass into dividing $Y$-state. Note that $P$ represents the total amount of metabolites obtained as result of the transformation of the nutrient substrate $S$. The two reproduction steps (RSR) describe the transfer of nutrient metabolite $P$ partially into $X$ - and $Y$-cells. The production step (RSP) describes the transition of the intermediate metabolites $P$ into (useful) product $P_{1}$ (EPS). The decay step (RSD) reflects the decay of $X$-cells assuming that the disintegrated cells transform into a waste product $Q$.

$$
\begin{aligned}
& S+X \stackrel{k_{1}}{\longrightarrow} Y \stackrel{k_{2}}{\longrightarrow} P+X, \\
& P+Y \stackrel{\beta}{\longrightarrow} 2 Y, \\
& P+Y \stackrel{\alpha}{\longrightarrow} X+Y, \\
& P+Y \stackrel{\gamma}{\longrightarrow} Y+P_{1}, \\
& X \stackrel{k_{d}}{\longrightarrow} Q
\end{aligned}
$$

Applying the mass action law to the above reaction steps as in chemical and enzyme kinetics we obtain the following system of ODE's where $p_{1}$ represents the product EPS:

$$
\begin{aligned}
& d s / d t=-k_{1} x s \\
& d x / d t=-k_{1} x s+k_{2} y+\alpha p y-k_{d} x \\
& d y / d t=k_{1} x s-k_{2} y+\beta p y \\
& d p / d t=k_{2} y-(\alpha+\beta+\gamma) p y \\
& d p_{1} / d t=\gamma p y .
\end{aligned}
$$




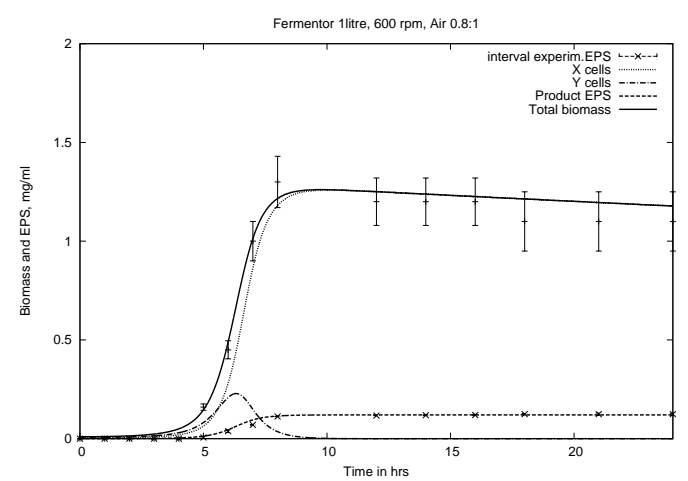

Fig. 4. Solution of model (2), $600 \mathrm{rpm}$

The values of the parameters numerically identified in model (2) are as follows: $k_{1}=5.8, k_{2}=$ $2.8, k_{d}=0.005, \alpha=9, \beta=9, \gamma=1.7, s(0)=$ $0.9, x(0)=0.011, y(0)=p(0)=p_{1}(0)=0$. The graphs of the solutions fitting the experimental data are visualized in Fig. 4. A very good fit of the biomass, as well as of the product EPS, can be observed.

Model (2) has been fitted for the measurement data obtained with other agitation rates. The results are always good within the accuracy of the experimental measurements. Figures 5 and 6 are examples of the results obtained for $500 \mathrm{rpm}$ and $100 \mathrm{rpm}$.

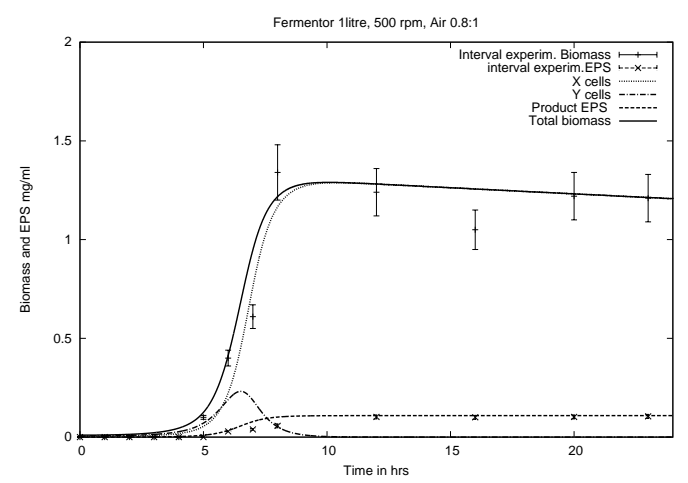

Fig. 5. Solution of model (2), $500 \mathrm{rpm}$

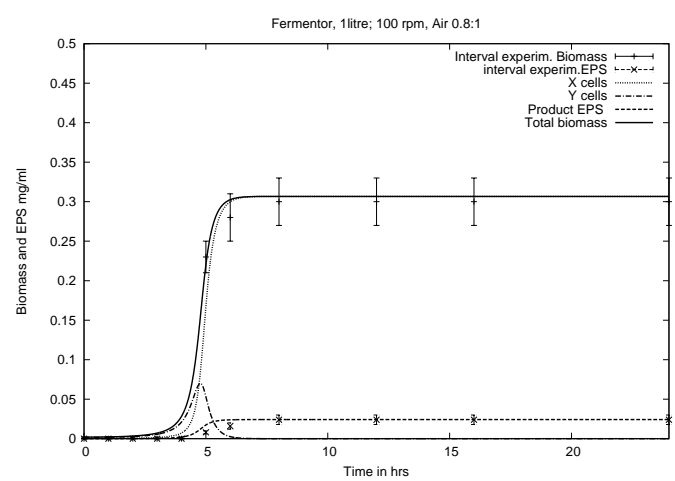

Fig. 6. Solution of model (2), $100 \mathrm{rpm}$

The coefficients obtained for the best fit at various agitation rates reported in Figure 11 are given in Table II.

\begin{tabular}{|c|ccccccc|}
\hline$r p m$ & $k_{1}$ & $k_{2}$ & $\alpha$ & $\beta$ & $\gamma$ & $s_{0}$ & $x_{0}$ \\
\hline 100 & 83 & 5.5 & 17 & 17 & 2.7 & 0.32 & 0.002 \\
400 & 13 & 2.8 & 9 & 9 & 1.7 & 0.6 & 0.01 \\
500 & 5.5 & 2.7 & 9 & 9 & 1.5 & 0.9 & 0.01 \\
600 & 5.8 & 2.8 & 9 & 9 & 1.7 & 0.9 & 0.01 \\
800 & 9 & 2.8 & 9 & 9 & 2.5 & 0.6 & 0.01 \\
\hline
\end{tabular}

TABLE II

COEFFICIENTS OF MODEL 22 FOR VARIOUS AGITATION RATES

It can be seen from Table $\amalg$ that the two coefficients $\alpha$ and $\beta$ are always equal. An interpretation could be that newborn cells belong wth equal probability to one of the two fractions (of dividing or non-dividing cells). Concerning the other coefficients $k_{1}, k_{2}, \gamma$, they have minimal values for 500$600 \mathrm{rpm}$. Note that these are the agitation rates corresponding to a maximum product synthesis.

For model (2) another "good" set of parameters is found to be the following $k_{1}=3, k_{2}=$ $1.4, \alpha=6.5, \beta=6.5, \gamma=1.2, k_{d}=0.005, s_{0}=$ $1.65, x_{0}=0.011, y_{0}=p_{0}=0$. The explanation of this phenomenon is the fact that the fitting of the mathematical solutions to the experimental data has been done with an optimization method 
which minimizes the sum of the squared differences between the experimental values and the mathematical values provided by the model (least square approximation) and the objective function has several local minima leading to several sets of parameters.

To conclude, Model (2) provides a very good representation of the experimental data. The model is a modification of an ODE model proposed in [1].

\section{Model 3}

Model 3 is a particular case of model (2). Here we assume that the rate constant $\alpha=0$, that is the first reproduction reaction step $P+Y \stackrel{\alpha}{\longrightarrow} X+Y$ is omitted. Consider then the following reaction scheme:

$$
\begin{aligned}
& S+X \stackrel{k_{1}}{\longrightarrow} Y \stackrel{k_{2}}{\longrightarrow} P+X, \\
& P+Y \stackrel{\beta}{\longrightarrow} 2 Y, \\
& P+Y \stackrel{\gamma}{\longrightarrow} Y+P_{1}, \\
& X \stackrel{k_{d}}{\longrightarrow} Q,
\end{aligned}
$$

It implies the following system of ODE's:

$$
\begin{aligned}
& d s / d t=-k_{1} x s \\
& d x / d t=-k_{1} x s+k_{2} y-k_{d} x \\
& d y / d t=k_{1} x s-k_{2} y+\beta p y \\
& d p / d t=k_{2} y-(\beta+\gamma) p y \\
& d p_{1} / d t=\gamma p y
\end{aligned}
$$

The values of the parameters in model (3) are identified as follows: $k_{1}=20, k_{2}=5, \beta=$ $1.5, \gamma=0.15, k_{d}=0.005 ; s_{0}=2.2, x_{0}=$ $0.02, y_{0}=0, p_{0}=p_{1}(0)=0$. The graphs of the solutions fitting real experimental data are visualized in Figure 77, The fit of the biomass and EPS to the experimental data is almost as good as in model (2). This shows that the reaction step $P+Y \stackrel{\alpha}{\longrightarrow} X+Y$ does not influence much the final solutions and can be omitted. The omission of this step can be interpreted as postulating that newborn cells are always in dividing state, which is biologically relevant [5]. Of course the division process can be inhibited in case that meanwhile environmental conditions become unfavorable. Model (3) is a modification of a model proposed in [10].

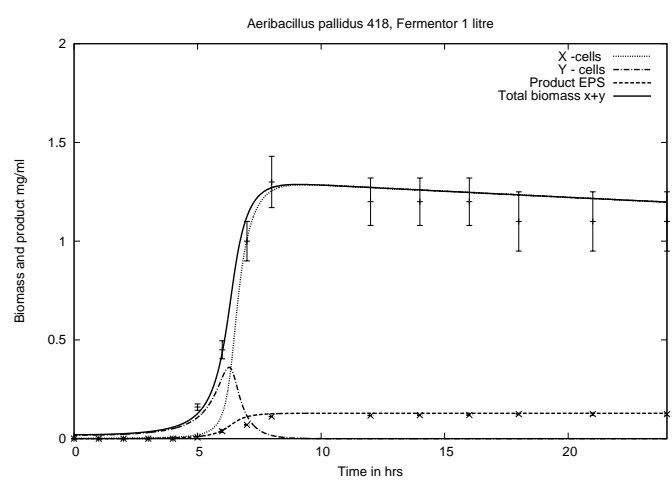

Fig. 7. Solution of model (3), $600 \mathrm{rpm}$

\section{Conclusion}

We described the dynamics of microbial growth and EPS synthesis using several mathematical models. The first model is a classical one, while models 2 and 3 are original structured models formulated in terms of reaction schemes, thereby the reaction growth step is borrowed from HenriMichaelis-Menten enzyme kinetics. As shown in [10] a reaction scheme using such a reaction step generalizes classical models like Model 1. The suggested models differ in the choice of the reaction steps. Compared to classical Model 1 the proposed models produce a better fit for the cell biomass experimental data.

To explain this, note that classical Monod type models make use of the so-called "specific growth rate function" $\mu(s)$. Recall that this function coincides (up to a multiplier) with the nutrient substrate uptake rate $\mu(s)$ in Michaelis-Menten enzyme kinetics. However, Michaelis-Menten substrate uptake function $\mu(s)$ is an approximation of the substrate rate function induced by the HenriMichaelis-Menten mechanism under the assumption that the enzyme/substrate ratio is small. The latter may not be true in vivo, as this ratio is not small in the cell cytoplasm [14], [15]. Note that our proposed models keep close to Henri-MichaelisMenten mechanism, which is independent on the 
value of the enzyme/substrate ratio.

Bacterial cells are metabolically active not only in the dividing state but in the non-dividing state as well [13]. Hence, when formulating reaction steps corresponding to these two states one should take into account such metabolic activities. Our dynamical models based on reaction schemes are first steps in this direction. We hope that the proposed models throw some light on the related cell growth mechanisms. Indeed, the reaction steps used in our models presume metabolic activity of the cell in its non-dividing state.

Our proposed models conform with the hypothesis from [10] saying that the reaction terms in cell growth dynamical systems can be deduced from appropriate biochemical reaction schemes with constant reaction rates via mass action law. Such an approach contributes to the interpretation of the underlying biological mechanism of the cell growth phenomena.

The biological experiments have been performed with highest possible precision in order to serve further for accurate numerical simulations. The overall goal is to verify as much as possible the reaction scheme approach for cell growth and product synthesis modelling and possibly determine a set of adequate reaction steps. A most useful feature of the proposed reaction scheme approach is its methodological value. This approach allows the biologists to focus on the model formulation in terms of reaction schemes as done in biochemistry; thus biologists may not need to formulate their models directly in terms of differential equations. Once the model is formulated in terms of reaction schemes, then the remaining part of the modelling process can be rather automated. Of course a theoretical (mathematical) study of the model, e.g. with respect to stability, may also be of scientific interest.

A most important conclusion (in our opinion) is that the modelling approach based on the subdivision of the cell population into two fractions (of dividing and non-dividing cells) proves as practically useful. This biological paradigm is different from the classical one based on the biomass phases (lag, log, etc) which implies simultaneous transitions of all cells from one biomass phase to the other. The classical approach is oriented towards properties of the whole bio-population, whereas our paradigm is oriented towards the individual cell cycles. Individual cell cycles are considered in [6], [7] under the hypothesis that a certain part of the cell lifetime is devoted to maturing and another part to division. This interpretation is in accord with recent findings of cell biology [5] and is confirmed by the proposed models.

We hope that our models contribute to a clarification of the biological mechanisms for the transition of an individual cell from a non-dividing to a dividing state (or conversely). Assume that the biomass has been inhibited or stressed for a sufficiently long period and (almost) all cells are in non-dividing state. When the environmental conditions become favorable, the cells start to divide, however this process does not happen simultaneously in time for all cells. The precise mechanism for this transition is not known; it is suggested that cell signalling (quorum sensing) plays an important role in this process. Some cells are able to quickly respond to environmental changes and then transmit signals to other cells. In certain time periods there exist significant numbers of both cell populations and this biologically realistic assumption is confirmed by the proposed models involving two cell fractions.

Future work. Future work is foreseen in the following directions: A. To study the biological relevance of the proposed reaction steps and the solutions of the corresponding dynamic systems; B. To consider continuous fermentation experimental data and appropriate modelling within the outlined methodology; C. To provide suitable fermentation experimental data for studying mathematically inhibition phenomena; D. To study mathematically the proposed dynamic systems with respect to stability. 


\section{ACKNOWLEDGMENT}

The authors are grateful to the National Fund for Scientific Research, Bulgaria, for financial support of this work (Contract DTK 02/46). They are also extremely grateful to both anonymous reviewers for their deep, detailed, very competent, constructive and beneficial comments and critical remarks.

\section{REFERENCES}

[1] Alt, R., S. Markov, Theoretical and computational studies of some bioreactor models, Computers and Mathematics with Applications 64 (2012), 350-360. http://dx.doi.org/10.1016/J.Camwa.2012.02.046

[2] Bastin G., D. Dochain, On-line Estimation and Adaptive Control of Bioreactors. Process measurement and control, Elsevier, 1990. http://dx.doi.org/10.1002/cite.330630220

[3] Burhan, N., Ts Sapundzhiev, V. Beschkov, Mathematical modelling of cyclodextrin-glucano- transferase production by immobilised cells of Bacillus circulans ATCC21783 at batch cultivation, Bioch. Eng. J. 35 (2007), 114-119.

[4] Kambourova, M., R. Mandeva, D. Dimova, A. Poli, B. Nicolaus, G. Tommonaro, Production and characterization of a microbial glucan, synthesized by Geobacillus tepidamans V264 isolated from Bulgarian hot spring, Carbohydr. Polym. 77 (2009), 338-343.

[5] Krebs, J. E., E. S. Goldstein, S. T. Kilpatrick, Lewin's GENES XI, Jones \& Bartlett Publ., 2014.

[6] Lemesle, V., J-L. Gouzé, A biochemically based structured model for phytoplankton growth in the chemostat, Ecological Complexity 2, 21-33 (2005). http://dx.doi.org/10.1016/j.ecocom.2004.08.00

[7] Lemesle, V., J.-L. Gouzé, A simple unforced oscillatory growth model in the chemostat, Bulletin of Mathematical Biology 70, 344-357 (2008). http://dx.doi.org/10.1007/s11538-007-9254-5

[8] Liu, C., L. Lu, Y. Liu, F. Wang, M. Xiao, Isolation, structural characterization and immunological activity of an exopolysaccharide produced by Bacillus licheni- formis 8-37-0-1, Biores. Technol., 101 (2010) 55285533.

[9] Markov, S., Biomathematics and interval analysis: a prosperous marriage. In: M. D. Todorov, Ch. I. Christov, Eds., AIP Conference Proceedings, Vol. 1301, Amitans2010, American Institute of Physics, 2010, 26-36. http://dx.doi.org/10.1063/1.3526621

[10] Markov, S., Cell Growth Models Using Reaction Schemes: Batch Cultivation, Biomath 2/2 (2013), 1312301. http://dx.doi.org/10.11145/j.biomath.2013.12.301

[11] Monod, J., The growth of bacterial cultures, Annual Reviews of Microbiology 3, 371-394 (1949). http://dx.doi.org/10.1146/annurev.mi.03.100149.002103

[12] Radchenkova, N., S. Vassilev, I. Panchev, G. Anzelmo, I. Tomova, B. Nicolaus, M. Kuncheva, K. Petrov, M. Kambourova, Production and properties of two novel exopolysaccharides synthesized by a thermophilic bacterium Aeribacillus pallidus 418, Applied Biochemistry and Biotechnology 171(1):31-43 (2013). http://dx.doi.org/10.1007/s12010-013-0348-2

[13] Rolfe, M. D. et al., Lag Phase Is a Distinct Growth Phase That Prepares Bacteria for Exponential Growth and Involves Transient Metal Accumulation, J. Bacteriol. 2012, 194(3):686. http://dx.doi.org/10.1128/JB.06112-11

[14] Schnell, S., Chappell, M. J., Evans, N. D., M. R. Roussel, The mechanism distinguishability problem in biochemical kinetics: The single-enzyme single-substrate reaction as a case study. C. $R$. Biologies 329, 51-61 (2006).

[15] Schnell, S., P. K. Maini, Enzyme kinetics at high enzyme concentration, Bulletin of Mathematical Biology 62, 483-499 (2000). http://dx.doi.org/10.1006/bulm.1999.0163

[16] Schnell, S., P. K. Maini, A century of enzyme kinetics: Reliability of the $K_{M}$ and $v_{\max }$ estimates, Comments on Theoretical Biology 8, 169-187 (2003). http://dx.doi.org/10.1080/08948550390206768

[17] Sissons, C. J., M. Cross, S. Robertson, A new approach to the mathematical modelling of biodegradation processes, Appl. Math. Modelling 10 (1986), 33-40. http://dx.doi.org/10.1016/0307-904X(86)90006-5 